Journal of Animal and Veterinary Advances 11 (6): 719-722, 2012

ISSN: $1680-5593$

(C) Medwell Journals, 2012

\title{
Relationship Between Fluoroquinolone Resistance and gyr , parC Gene in Streptococcus suis Type 2 Isolates in Hebei Province China
}

\author{
Ping Rui, Zengjun Ma, QiuYue Wang, Hai Fang, JianHua Wu, \\ ZhiXin Fu, Qing Hui Jia and Xuexia Wen \\ Key Laboratory of Preventive Veterinary Medicine of Hebei, \\ Hebei Normal University of Science and Technology, 066004 Qinhuangdao, China
}

\begin{abstract}
To detect the relationship between fluoroquinolone resistance and gyrA, parC gene mutation in Streptococcus suis type 2. The MIC of 14 strains of Streptococcus suis type 2 were determined by microdilution. The genes encoding the Quinolone-Resistance Determining Region (QRDRs) of parC and gyrA in fluoroquinolone-susceptible and resistant Streptococcus suis clinical isolates were identified and sequenced. Ser to Phe and Arg to Leu mutation at position 79 and 87 of the parC gene was detected in fluoroquinolone resistance SS2 and Arg to Ser or Ser to Arg mutations at the position 66 or 81 of gyrA gene were detected in 2 highly resistance strains; no amino acid changes in gyrA or parC were detected for 7 fluoroquinolonesusceptible strains; the mutations in both genes were found in the strains with $\mathrm{MIC}$ of fluoroquinolone $>32 \mu \mathrm{g} \mathrm{mL}^{-1}$. Mutations in parC gene may result in low level resistance against fluoroquinolone and mutations in both gyrA and parC genes result in high level resistance.
\end{abstract}

Key words: Streptococcus suis, fluoroquinolone, parC gene, gyrA gene, amino acid, China

\section{INTRODUCTION}

Streptococcus suis, a class of swine diseases caused by streptococcal bacteria has occurred all over the world. Some pathogenic streptococci are important zoonotic pathogens that can lead to a variety of suppurative diseases, pneumonia, meningitis, endocarditis, septicemia and septic arthritis which caused great harm to human and animal health (Kongwang and Chengping, 2000; Najati and Saboktakin, 2010; Seyed et al., 2011). Its high morbidity and mortality caused severe economic losses to the porcine industry. In recent years with the inappropriate use of antibiotics, Streptococcus suis is also experienced varying degrees of antibiotic resistance including fluoroquinolone resistance. At present, it is only reported that parC, gyrA gene mutations of Streptococcus suis separated from of the Northeast region of China associate with drug resistance (Yuan et al., 2009). Nucleotide and amino acid mutations of parC, gyr $A$ gene of fluoroquinolone-resistant Streptococcus suis separated from Hebei province of China were deteced on this study and the relationship between these mutations in parC, gyrA gene and the different degrees of resistance was explained.

\section{MATERIALS AND METHODS}

Origin of strain: The Streptococcus suis type 2 strains were separated from lungs, spleen, kidney, heart, brain and synovial fluid in pigs with typical infection of Streptococcus suis in Qinhuangdao, Tangshan, Zhangjiakou, Shijiazhuang, Handan of Hebei province during 2005-2010. About 14 strains were obtained by PCR identification. Staphylococcus aureus ATCC29213 as the quality control strain were purchased from Hangzhou Tianhe Microorganism Reagent Co., Ltd.

Medium and reagents: $M H$ mediums were purchased from Hang zhou Tianhe Microorganism Reagent Co., Ltd. and $5 \%$ calf serum was added to it before usage; drug standards were purchased from China Institute of Veterinary Drugs Control; Taq DNA Polymerase (plus) $\left(2 \mathrm{U}_{\mu} \mathrm{L}^{-1}\right)$, DNA Marker DL2000 (60 ng $\left.\mu \mathrm{L}^{-1}\right)$ were from TaKaRa Biotechnology Co., Ltd. Goldview dye, centrifugal cylindrical agarose gel, DNA purification kit Dye, Agarose Gel DNA Extraction kit (Centrifugal columnar) purchased from Tiangen Biotech (Beijing) Co., Ltd.

Primers: Primers were designed accroding to the reference (Escudero et al., 2007). The primers of gyrA were 5'-CGCCGTATTTTGTATGGGATG-3' and 5'GTTCCGTTAACCAGAAGGTT-3'. The primers of parC were 5'-AAGGACGGCAACACTTTTGAC-3' and 5'AGTGGGTTCTTTTTCCGTATC-3'. Product length was 377 and 312 bp while annealing temperature 48.9 and $42.7^{\circ} \mathrm{C}$, respectively. The fragments were amplified and then sequenced Sangon Biotech (Shanghai) Co., Ltd.

Corresponding Author: Zengjun Ma, Key Laboratory of Preventive Veterinary Medicine of Hebei, Hebei Normal University of Science and Technology, 066004 Qinhuangdao, China 
Drug sensitivity test in vitro: About 14 strains of SS2 were measured the Minimum Inhibitory Concentration (MIC) to 4 fluoroquinolones including Ciprofloxacin $(\mathrm{CPF})$, Enrofloxacin (ENR), Ofloxacin (OFL) and Levofloxacin (LVX). The specific operation and the results were according to NCCLS standards (M31-A2. Wayne, USA. 2000) (National Committee for Clinical Laboartory Standards, 2000).

Preparation of PCR template: About 7 resistant strains and 7 susceptible strains were selectd. The colonies on rabbit blood agar plates were transferred to a containing $5 \%$ calf serum Streptococcus medium and grown at $37^{\circ} \mathrm{C}$ overnight. The $1 \mathrm{~mL}$ preparation in $\mathrm{EP}$ tube was centrifuged for $1 \mathrm{~min}$ at $12,000 \mathrm{rpm}$ to remove supernatant the cellular debris, then resuspended in $200 \mu \mathrm{L}$ deionized water. After boiled for $10 \mathrm{~min}$, the strains were centrifuged for $3 \mathrm{~min}$ at $12000 \mathrm{rpm}$. Finally, the supernatant without precipitation were stored in $-20^{\circ} \mathrm{C}$ as the PCR template.

gyrA, parC gene detection: Amplification was performed in a total volume of $25 \mu \mathrm{L}$ containing $2.0 \mu \mathrm{L}$ dNTP mixture (each $2.5 \mathrm{mM}), 1.0 \mu \mathrm{L}$ each primer $\left(10 \mathrm{pmol} \mu \mathrm{L}^{-1}\right), 0.5 \mu \mathrm{L}$ ExTaq DNA polymerase $(1.25 \mathrm{U}), 2.5 \mu \mathrm{L} 10 \times \mathrm{PCR}$ buffer $\left(\mathrm{Mg}_{2}+\right.$ Plus), $2.5 \mu \mathrm{L}$ DNA template and distilled water. The PCR assay comprised preincubation at $94^{\circ} \mathrm{C}$ for $5 \mathrm{~min}$ followed by 30 cycles of $94^{\circ} \mathrm{C} 1 \mathrm{~min}, 48.9 / 42.7^{\circ} \mathrm{C} 50 \mathrm{sec}$ and $72^{\circ} \mathrm{C} 1 \mathrm{~min}$. A final extension was performed at $72^{\circ} \mathrm{C}$ for $10 \mathrm{~min}$. The PCR products were visualized by electrophoresis on a $1 \%$ agarose gel by standard procedures.

gyrA, parC gene recovery and sequencing: The $\mathrm{PCR}$ products, 312 bp gyrA gene fragment and 377 bp parC gene fragment, purified by gel, then were recovered and sequenced. Sequencing was performed by Sangon Biotech (Shanghai) Co., Ltd. and the sequence data were analyzed using BLAST of GenBank and BioEdit Software.

\section{RESULTS AND DISCUSSION}

Fluoroquinolones against strains of $S$. suis isolated antibacterial activities in vitro: For selected ciprofloxacin, ofloxacin, enrofloxacin and levofloxacin four fluoroquinolone antibacterial drugs, 14 strains of SS2 isolates inhibitory rate were $50(7 / 14), 50(7 / 14), 50(7 / 14)$ and $57.1 \%(8 / 14)$, respectively. The MIC values of 7 highly resistance strains and 7 susceptible strains were shown in Table 1.

Reasults of $\operatorname{parC}$ and gyrA gene amplification: About 14 strains of SS2 isolates were amplified using chromosomal DNA as temple, the fragment of parC and gyrA gene were deteced, consistence with the expected size of 312 (Fig. 1) and 377bp (Fig. 2).

\begin{tabular}{lrrrr}
\multicolumn{5}{l}{ Table 1: Activities in vitro of selected fluoroquinolones for SS2 isolated } \\
\hline \multicolumn{5}{c}{ MIC $\left(\mu \mathrm{g} \mathrm{mL} \mathrm{mL}^{-1}\right)$} \\
Strains & CPF & OFL & ENR & LVX \\
\hline 1 & 256.000 & 256.0 & 128.00 & 128.00 \\
2 & 128.000 & 128.0 & 64.00 & 64.00 \\
3 & 16.000 & 16.0 & 16.00 & 8.00 \\
4 & 32.000 & 16.0 & 16.00 & 16.00 \\
5 & 32.000 & 16.0 & 32.00 & 16.00 \\
6 & 64.000 & 32.0 & 16.00 & 16.00 \\
7 & 16.000 & 8.0 & 8.00 & 1.00 \\
8 & 0.250 & 0.5 & 0.50 & 0.50 \\
9 & 0.125 & 0.5 & 0.25 & 0.25 \\
10 & 0.500 & 1.0 & 0.50 & 1.00 \\
11 & 0.500 & 1.0 & 0.50 & 1.00 \\
12 & 0.500 & 1.0 & 0.50 & 1.00 \\
13 & 0.500 & 1.0 & 0.50 & 0.50 \\
14 & 0.500 & 1.0 & 0.50 & 1.00 \\
\hline
\end{tabular}

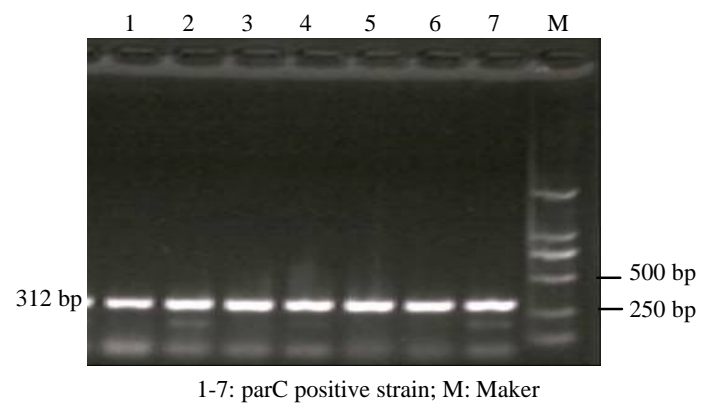

Fig. 1: PCR amplification of parC of chromosomalDNA in $\mathrm{SS} 2$

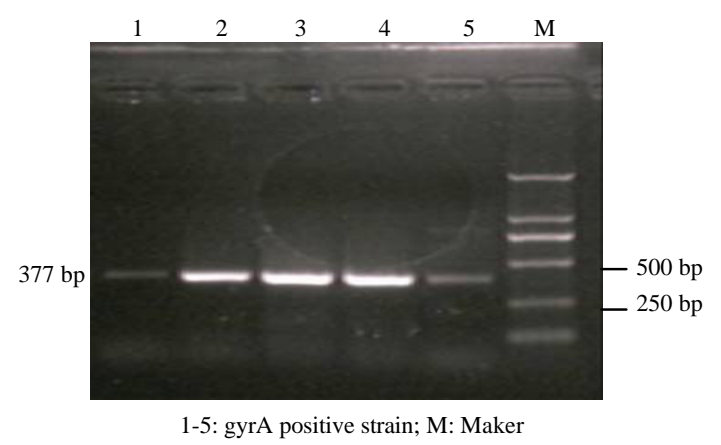

Fig. 2: PCR amplification of gyrA of chromosomal DNA in $\mathrm{SS} 2$

The amino acid sequence analysis of $\operatorname{par} C$ gene: Compared with the reference sequence AB287006.1, the mutations on the amino acid sequence of parC about 7 drug-resistant strains are less. There are 2 strains with Ser $79 \rightarrow$ Phe, 1 strains with Arg87 $\rightarrow$ Leu in the Quinolone Resistance-Determining Regions (QRDRs) of the parC gene in streptococci and 3 strains occurred Met1 $26 \rightarrow \mathrm{Val}$ beyond the QRDR. The other one is no mutations (Fig. 3).

The amino acid sequence analysis of gyr $A$ gene: Compared with the reference sequence AB081330.1., 


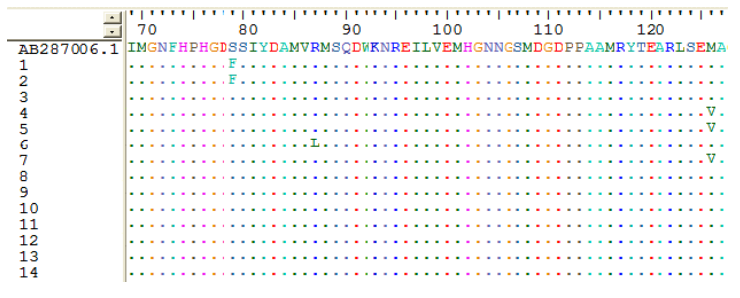

Fig. 3: Comparison of amino acid sequence of parC (fragments involving mutational sites)

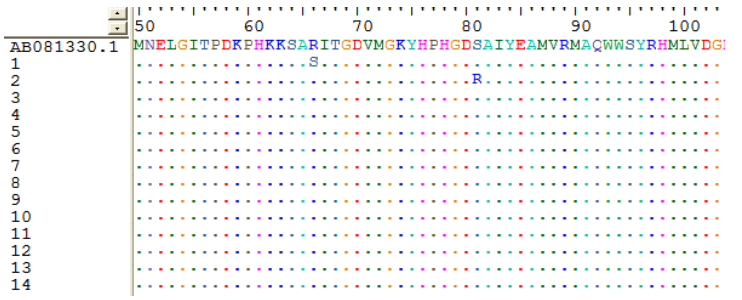

Fig. 4: Comparison of amino acid sequence of gyrA (fragments involving mutational sites)

result were showed. One strains presented Arg $66 \rightarrow$ Ser, 1 strains presented Ser $81 \rightarrow$ Arg in the Quinolone ResistanceDetermining Regions (QRDRs) of the gyrA gene in streptococci. There were six resistant strains without mutations being found (Fig. 4).

From the sensitivity test results, 14 strains of Streptococcus suis isolates showed significant crossresistance to ciprofloxacin, enrofloxacin, ofloxacin and levofloxacin. The resistance rate was $42.9 \sim 50 \%$. The number of high-leval resistant strains is increasing year by year. With the rapid development of economy and the increaseing use of antibiotics in the prevention of swine diseases, resistance of Streptococcus suis has been so widespread and severe, researchers should pay more attention to the clinical treatment process.

The mutations in amino acid sequence of gyrA gene about 14 strains of Streptococcus suis are not much. In the Quinolone Resistance-Determining Regions (QRDRs) of the gyrA gene in Streptococcus suis Arg66 $\rightarrow$ Ser and Ser81 $\rightarrow$ Arg in the study which are inconsistent with the previous report (Yuan et al., 2009, Escudero et al., 2007) with Ser81 $\rightarrow$ Lys, Ser81 $\rightarrow$ Phe, Ser81 $\rightarrow$ Tyr, Ser81 $\rightarrow$ Ile in foreign reports and Ser81 $\rightarrow \mathrm{Val}, \mathrm{G} \ln \rightarrow \mathrm{Arg}$. These two mutations have not been reported. There are 2 strains with Ser $79 \rightarrow$ Phe, 1 strains with Arg87 $\rightarrow$ Leu in the Quinolone Resistance-Determining Regions (QRDRs) of the parC gene in 7 resistant strains of streptococci and 3 strains presented Met126 $\rightarrow$ Val beyond the QRDR. No mutation in 1 resistant strain was found. Ser $79 \rightarrow$ Phe is consistent with reports (Escudero et al., 2007) and differs from Ser80, Ile 70, G1y78 and Leu89 reported (Yuan et al., 2009).

\section{CONCLUSION}

This study shows that it was found that the QRDR of $\operatorname{par} C$ and gyrA gene were detected no mutations when Streptococcus suis had MICs to ciprofloxacin and enrofloxacin of $<1 \mu \mathrm{g} \mathrm{mL} \mathrm{L}^{-1}$ to Ofloxacin and levofloxacin of $<2 \mu \mathrm{g} \mathrm{mL}{ }^{-1}$. With MICs of $\geq 8 \mu \mathrm{g} \mathrm{mL} \mathrm{m}^{-1}$, mutations Ser $79 \rightarrow$ Phe, Arg $87 \rightarrow$ Leu, Met $126 \rightarrow$ Val were presented in $\operatorname{par} C$ gene, of which Ser $79 \rightarrow$ Phe is the most common. The strains with MICs of $32 \mu \mathrm{g} \mathrm{mL}^{-1}$ presented Ser $79 \rightarrow$ Phe in ParC subunite and Arg66 $\rightarrow$ Ser, Ser81 $\rightarrow$ Arg in gyrA subunite. It was confirmed that the changes of single topoisomerase IV caused low-level resistance to quinolones in Streptococcus suis while the high-level resistance is the result of the changes of topoisomerase IV and DNA gyrase (Davies et al., 1999; Varon et al., 1999). It was also shown that some drug-resistant strains were no found amino acid mutations in parC and gyrA genes. Whether fluoroquinolone resistance in Streptococcus suis was caused by efflux mechanism (Wang et al., 2007) and plasmid-mediated horizontal transmission mechanism (Hopkins et al., 2007) or not still for further study.

\section{ACKNOWLEDGEMENTS}

This study were financially supported by Natural funded project of Hebei (C2009000877), Department of Education Project of Hebei (2008448), Hebei Science and Technology Support Program (09220402D).

\section{REFERENCES}

Davies, T.A., G.A. Pankuch, B.E. Dewasse, M.R. Jacobs and P.C. Appelbaum, 1999. In vitro development of resistance to five quinolones and amoxicillinclavulanate in Streptococcus pneumoniae. Antimicrob. Agents Chemother., 43: 1177-1182.

Escudero, J.A., A.S. Millan, A. Catalan, A.G. de la Campa and E. Rivero et al., 2007. First characterization of fluoroquinolone resistance in Streptococcus suis. Antimicrob. Agents Chemother, 51: 777-782.

Hopkins, K.L., L. Wootton, M.R. Day and E.J. Threlfall, 2007. Plasmid mediated quinolone resistance determinant qnrS1 found in Salmonella enteric strains isolated in the UK. J. Antimicrob. Chemother., 59: 1071-1075.

Kongwang, H.E. and L. Chengping, 2000. Pathopoiesis and virulence genes of Streptococcus suis serotypes 2. Vet. Sci. China, 30: 17-18. 
Najati, N. and L. Saboktakin, 2010. Prevalence and underlying etiologies of neonatal hypoglycemia. Pak. J. Biol. Sci., 13: 753-756.

National Committee for Clinical Laboartory Standards, 2000. Perfomrance Standards for Antimicrobial Disk and Dilution Susceptibility Test for Bacteria Isolated from Animals: Approved Standard. NCCLS document M31-A2, Wayne,USA.

Seyed, Z.B., S.S. Tabrizi and G.R. Abdollahpour, 2011. Stomatocytosis in the ruminent: A first report. Asian J. Anim. Vet. Adv., 63: 1089-1093.
Varon, E., C. Janoir, M.D. Kitzis and L. Gutmann, 1999. ParC and GyrA may be interchangeable initial targets of some fluoroquinolones in Streptococcus pneumoniae. Antimicrob. Agents Chemother., 43: 302-306.

Wang, D.D., T.Y. Sun and Y.J. Hu, 2007. Contributions of efflux pumps to high level resistance of Pseudomonas aeruginosa to ciprofloxacin. Chin. Med. J., 120: 68-70.

Yuan, Q., Q. Wang and X.Y. Li, 2009. Relationship between ciprofloxa cinres is tance and gyrA, parC gene mutation in Streptococcus suis. Chin. J. Prev. Vet. Med., 31: 201-203. 